\title{
An Integral Sliding Mode Control of Uncertain Chaotic Systems via Disturbance Observer
}

\author{
Hua Zhang (10 \\ Zhengzhou Preschool Education College, Zhengzhou 450099, China \\ Correspondence should be addressed to Hua Zhang; zhanghua27@163.com
}

Received 1 January 2021; Revised 4 February 2021; Accepted 13 February 2021; Published 25 February 2021

Academic Editor: Heng Liu

Copyright $\odot 2021$ Hua Zhang. This is an open access article distributed under the Creative Commons Attribution License, which permits unrestricted use, distribution, and reproduction in any medium, provided the original work is properly cited.

\begin{abstract}
This paper proposes an integral sliding mode control (ISMC) method of a class of uncertain chaotic systems with saturation inputs. Firstly, fuzzy logic system (FLS) is used to estimate the unknown nonlinear function. Then, a disturbance observer is constructed to estimate a compound disturbance, which contains the external disturbance, the error of saturation input and control output, and the fuzzy estimation error. Subsequently, a proposed integral sliding mode controller can ensure that all signals of the closed-loop system are ultimately bounded, and based on the dynamic system of the integral sliding mode variable itself, the ultimate bound of the tracking error can be estimated. Simulation results show that the proposed ISMC method is more effective than the traditional ISMC method.
\end{abstract}

\section{Introduction}

Due to the unpredictability and the sensitivity to initial conditions, the chaotic system makes itself useful in many fields such as information processing, secure communications, and mechanical systems $[1,2]$. However, some actual systems, such as flutter of aircraft wings and vibration of horizontal platforms, may cause bad results if the chaotic behavior occurs. Therefore, it is necessary to suppress such harmful chaotic behavior. At present, many methods have been proposed to stabilize or synchronize chaotic systems, such as adaptive control method $[3,4]$, backstepping control $[5,6]$, impulsive control [7], intermittent control [8], and sliding mode control [9-14].

As we all know, the advantage of using sliding mode control method to control chaotic systems and other nonlinear systems is its good robustness. For example, Wang et al. [11] proposed a nonsingular terminal SMC method to ensure that all states of the chaotic system reach the designed sliding surface in finite time. By using adaptive terminal SMC, Yang and $\mathrm{Ou}$ [12] studied the synchronization problem of chaotic gyros. Similarly, by employing adaptive SMC, Chen et al. [13] investigated the synchronization for multiple uncertain coupled chaotic systems. It can be seen that the abovementioned sliding mode control method only considers whether the arrival condition of the sliding surface can be satisfied and does not consider the estimation problem for system uncertainties. Meanwhile, the hysteresis of control switching will cause chattering phenomenon. In order to eliminate this disadvantage, Haghighi and Mobayen [15] proposed a high-order terminal SMC technique for a class of fourth-order systems. However, the higher-order sliding mode controller includes larger amount of higherorder derivatives of sliding mode variable, which may lead to increased noise in closed-loop systems.

Recently, in order to estimate system uncertainties or external disturbances, Zhou et al. [16-20] explored the construction principle of disturbance observer and combined different control methods to achieve the stability of closed-loop systems. Based on the disturbance observer and fuzzy terminal SMC, Vahidi-Moghaddam et al. [17] investigated the finite-time asymptotic stability of uncertain MIMO systems. $\mathrm{Xu}$ in [18] proposed composite terminal SMC learning control schemes for quadrotor dynamics via the disturbance observer. However, control results of [17-20] only ensure that all signals of the closed-loop system approach a small region, but the ultimate bound of this area has not been estimated. 
Inspired by the abovementioned works, this paper investigates the problem of integral sliding mode control for a class of uncertain chaotic systems with saturation inputs, and the proposed integral SMC method can not only guarantee all signals of the closed-loop system are bounded but also accurately estimate system uncertainties. The main contributions of this paper are summarized as follows:

(1) Compared with the traditional integral sliding mode variable, the integrated sliding mode variable proposed in this paper can estimate the error bound by its own dynamics when the boundedness of the integrated sliding mode variable is guaranteed.

(2) The established disturbance observer and fuzzy parameter law can accurately estimate system uncertainties.

(3) Compared with the traditional ISMC method, the proposed method in this paper can eliminate the chattering phenomenon.

The rest of this work is arranged as follows. Some assumptions and lemmas and the problem statement are presented in Section 2. Section 3 gives sliding mode control design and stability analysis. In Section 4, some comparison results are presented to show the validity of the proposed method. At last, the conclusion is included in Section 5.

\section{Preliminaries}

In general, the chaotic system can be described as

$$
\dot{\mathbf{x}}=\mathbf{F}(\mathbf{x})
$$

where $\mathbf{x}=\left[x_{1}, x_{2}, \ldots, x_{n}\right]^{T} \in R^{n}$ is the state vector and $\mathbf{F}(\mathbf{x})=\left[f_{1}(\mathbf{x}), f_{2}(\mathbf{x}), \ldots, f_{n}(\mathbf{x})\right]^{T}$ is the nonlinear vector function. Many classic chaotic systems such as Lorenz chaotic system, Chen chaotic system, and Lü chaotic system can be abbreviated as the form of system (1), and the controlled chaotic system (1) is expressed as

$$
\dot{\mathbf{x}}=\mathbf{F}(\mathbf{x})+\mathbf{d}(t)+\operatorname{sat}(\mathbf{u}),
$$

where $\mathbf{d}(\mathbf{t})=\left[d_{1}(t), d_{2}(t), \ldots, d_{n}(t)\right]^{T}$ is the external disturbance vector, $\mathbf{u}=\left[u_{1}, u_{2}, \ldots, u_{n}\right]^{T}$ is the control output vector, and $\operatorname{sat}(\mathbf{u})=\left[\operatorname{sat}\left(u_{1}\right) \text {, sat }\left(u_{2}\right), \ldots, \operatorname{sat}\left(u_{n}\right)\right]^{T}$ is the control input vector subject to saturation type nonlinearity, and $\operatorname{sat}\left(u_{i}\right)$ is described as

$$
\operatorname{sat}\left(u_{i}\right)= \begin{cases}u_{i}^{*} \cdot \operatorname{sign}\left(u_{i}\right), & \text { if }\left|u_{i}\right| \geq u_{i}^{*}, \\ u_{i}, & \text { if }\left|u_{i}\right|<u_{i}^{*},\end{cases}
$$

where $u_{i}^{*}$ is the unknown bound of symmetric input saturation.

Define the tracking error vector $\mathbf{e}=\mathbf{x}-\mathbf{x}_{\mathbf{d}}=\left[e_{1}, e_{2}, \ldots\right.$, $\left.e_{n}\right]^{T}$, and $\mathbf{x}_{\mathbf{d}}=\left[x_{d 1}, x_{d 2}, \ldots, x_{d n}\right]^{T}$ is the reference signal. According to (2), we obtain the following error dynamic system:

$$
\dot{\mathbf{e}}=\mathbf{F}(\mathbf{x})+\mathbf{d}(t)+\operatorname{sat}(\mathbf{u})-\dot{\mathbf{x}}_{\mathbf{d}} .
$$

In order to achieve effective tracking of the reference signal $\mathbf{x}_{\mathbf{d}}$ by the state $\mathbf{x}$, the following assumptions about $\mathbf{F}(\mathbf{x}), \mathbf{x}_{\mathbf{d}}$, and $\mathbf{d}(t)$ are made.

Assumption 1. The nonlinear function $\mathbf{F}(\mathbf{x})$ is unknown but bounded, and the state $\mathbf{x}$ is measurable.

Assumption 2. The reference signal $\mathbf{x}_{\mathbf{d}}$ is $\mathscr{C}^{2}$, and $\mathbf{x}_{\mathbf{d}}$ and $\dot{\mathbf{x}}_{d}$ are available.

Assumption 3. The time-varying external disturbance $\mathbf{d}(t)$ and its derivative are unknown but bounded.

Define $\Delta \mathbf{u}=\left[\Delta u_{1}, \Delta u_{2}, \ldots, \Delta u_{n}\right]^{T} \in R^{n}$, where $\Delta u_{i}=$ sat $\left(u_{i}\right)-u_{i}, i=1,2, \ldots, n$.

Remark 1. For the error system (4), we can use method [21] to track the reference signal $\mathbf{x}_{\mathbf{d}}$. Firstly, let $\mathbf{F}(\mathbf{x})=\overline{\mathbf{F}_{1}(x)}+\Delta \mathbf{F}$, where $\overline{\mathbf{F}(x)}$ is known. Define $\mathbf{D}(t)=\mathbf{d}(t)+\Delta \mathbf{u}+\Delta \mathbf{F}$, and assume that $\|\dot{\mathbf{D}}\| \leq \delta$, where $\delta$ is unknown. Then, construct the disturbance observer $\widehat{\mathbf{D}}$ as $\widehat{\mathbf{D}}=\widehat{\eta}+\mathbf{K}_{1} \mathbf{e}$, where $\widehat{\eta}$ is the intermediate variable and satisfies

$$
\dot{\hat{\eta}}=-\mathbf{K}_{1}\left(\overline{\mathbf{F}_{1}(x)}+\mathbf{u}+\widehat{\eta}+\mathbf{K}_{1} \mathbf{e}\right),
$$

where $\mathbf{K}_{1}=\operatorname{diag}\left(k_{11}, \ldots, k_{1 n}\right), k_{1 j}$ is the positive constant, and design the following control law $\mathbf{u}$ as

$$
\mathbf{u}=-\overline{\mathbf{F}_{1}(x)}-\widehat{\mathbf{D}}-\mathbf{K}_{2} \mathbf{e},
$$

where $\mathbf{K}_{2}=\operatorname{diag}\left(k_{21}, \ldots, k_{2 n}\right), k_{2 j}, j=1,2, \ldots, n$ are the positive constants. This method can guarantee that $\mathbf{e}$ and $\widetilde{\mathbf{D}}$ are uniformly bounded. However, this method does not cover two situations: one is that the function $\mathbf{F}(\mathbf{x})$ is unknown; the other is whether $\mathbf{F}(\mathbf{x})+\mathbf{D}$ can be accurately estimated. In order to consider the abovementioned two situations, this paper will use the integral sliding mode control method combined with the disturbance observer and fuzzy logic technology to design a control method to realize the fast stability of tracking error $\mathbf{e}(t)$. In addition, the controller and error state will effectively eliminate chattering phenomenon.

Define $\bar{f}_{i}(\mathbf{x})=l_{i} f_{i}(\mathbf{x})$, where $l_{i}$ is the positive design constant. By using FLSs, $\bar{f}_{i}(\mathbf{x})$ can approximate as

$$
\bar{f}_{i}(\mathbf{x})=\theta_{\bar{f}_{i}}^{* T} \varphi_{\bar{f}_{i}}(\mathbf{x})+\varepsilon_{\bar{f}_{i}}, \quad\left|\mathcal{\varepsilon}_{\bar{f}_{i}}\right| \leq \varepsilon_{i}^{M},
$$

where $\theta_{\bar{f}_{i}}^{*} \in R^{m_{i}}$ are the ideal weight vectors, $\varepsilon_{\bar{f}}$ is the approximation error, and $\varepsilon_{i}^{M}$ is the upper bound of $\varepsilon_{\bar{f}_{i}} \cdot \varphi_{\bar{f}_{i}}$ $(\mathbf{x})=\left[\varphi_{\bar{f}_{i 1}}(\mathbf{x}), \varphi_{\bar{f}_{i 2}}(\mathbf{x}), \ldots, \varphi_{\bar{f}_{i m_{i}}}(\mathbf{x})\right]^{T} \in R^{m_{i}}$, where $\varphi_{\bar{f}_{i,}}(\mathbf{x})$, $j=1, \ldots, m_{i}$ are the Gaussian functions. Obviously, there exists a positive constant $p^{*}$ such that $\left\|\varphi_{\bar{f}_{i}}(\mathbf{x})\right\| \leq p^{*}$.

Define $\widetilde{\theta}_{\bar{f}_{i}}=\theta_{\bar{f}_{i}}^{*}-\widehat{\theta}_{\bar{f}_{i}}$, where $\widehat{\theta}_{\bar{f}_{i}}$ is the estimation of $\theta_{f_{i}}^{*}$. So, we obtain

$$
f_{i}(\mathbf{x})=l_{i}^{-1} \bar{f}_{i}(\mathbf{x})=l_{i}^{-1} \tilde{\theta}_{f_{i}}^{T} \varphi_{\bar{f}_{i}}(\mathbf{x})+l_{i}^{-1} \widehat{\theta}_{\bar{f}_{i}}^{T} \varphi_{\bar{f}_{i}}(\mathbf{x})+l_{i}^{-1} \varepsilon_{\bar{f}_{i}}(\mathbf{x}) .
$$


Denote $\mathbf{L}^{-1} \widetilde{\theta}_{\mathbf{F}}^{T} \varphi_{\mathbf{F}}(\mathbf{x})=\left[l_{1}^{-1} \widetilde{\theta}_{\bar{f}_{1}}^{T} \varphi_{\bar{f}_{1}}(\mathbf{x}), l_{2}^{-1} \widetilde{\theta}_{f_{2}}^{T} \varphi_{\bar{f}_{2}}(\mathbf{x}), \ldots\right.$, $\left.l_{n}^{-1} \widetilde{\theta}_{\bar{f}_{n}}^{T} \varphi_{\bar{f}_{n}}(\mathbf{x})\right]^{T}, \mathbf{L}^{-1} \hat{\theta}_{\mathbf{F}}^{T} \varphi_{\mathbf{F}}(\mathbf{x})=\left[l_{1}^{-1} \widehat{\theta}_{\bar{f}_{1}}^{T} \varphi_{\bar{f}_{1}}(\mathbf{x}), l_{2}^{-1} \widehat{\theta}_{f_{2}}^{T} \varphi_{\bar{f}_{2}}(\mathbf{x})\right.$, $\left.\ldots, l_{n}^{-1} \widehat{\theta}_{\bar{f}_{n}}^{T} \varphi_{\bar{f}_{n}}(\mathbf{x})\right]^{T}, \mathbf{L}^{-1} \varepsilon_{\mathbf{F}}(\mathbf{x})=\left[l_{1}^{-1} \varepsilon_{\bar{f}_{1}}(\mathbf{x}), l_{2}^{-1} \varepsilon_{\bar{f}_{2}}(\mathbf{x}), \ldots, l_{n}^{-1}\right.$ $\left.\varepsilon_{\bar{f}_{n}}(\mathbf{x})\right]^{T}$. So, $\mathbf{F}(\mathbf{x})$ in (4) can be expressed as

$$
\mathbf{F}(\mathbf{x})=\mathbf{L}^{-1} \tilde{\theta}_{\mathbf{F}}^{T} \varphi_{\mathbf{F}}(\mathbf{x})+\mathbf{L}^{-1} \widehat{\theta}_{\mathbf{F}}^{T} \varphi_{\mathbf{F}}(\mathbf{x})+\mathbf{L}^{-1} \varepsilon_{\mathbf{F}}(\mathbf{x}) .
$$

Let $\mathbf{D}=\left[D_{1}, D_{2}, \ldots, D_{n}\right]^{T}=\mathbf{L}^{-1} \varepsilon_{\mathbf{F}}(\mathbf{x})+\mathbf{d}(t)+\Delta \mathbf{u}$. The error system (4) can be rewritten as

$$
\dot{\mathbf{e}}=\mathbf{L}^{-1} \tilde{\theta}_{\mathbf{F}}^{T} \varphi_{\mathbf{F}}(\mathbf{x})+\mathbf{L}^{-1} \hat{\theta}_{\mathbf{F}}^{T} \varphi_{\mathbf{F}}(\mathbf{x})+\mathbf{u}+\mathbf{D}-\dot{\mathbf{x}}_{\mathbf{d}} .
$$

Because the compounded disturbance $\mathbf{D}$ is unknown, it cannot appear directly in the controller design. But from a practical system point of view, D is clearly bounded. Combining with the structural ideas of disturbance observer $[18,21,22]$, we also make the following assumption.

Assumption 4. The derivative of $\mathbf{D}$ satisfies $\|\dot{\mathbf{D}}\| \leq D^{*}$, where $D^{*}$ is an unknown positive constant.

The following lemmas are introduced for the subsequent discussions.

Lemma 1 (see [23]). Let $0<p_{1}<p_{2}$, then

$$
\left(\sum_{i=1}^{n}\left|a_{i}\right|^{p_{2}}\right)^{\left(1 / p_{2}\right)} \leq\left(\sum_{i=1}^{n}\left|a_{i}\right|^{p_{1}}\right)^{\left(1 / p_{1}\right)},
$$

where $a_{i}$ is the real number.

Lemma 2 (see [24]). For any $z \in R, 1>\gamma \geq 0$, and $\epsilon>0$, the following inequality holds:

$$
|z|^{\gamma+1}-z|z|^{\gamma} \tan h\left(\frac{z}{\epsilon}\right) \leq \eta_{\gamma} \epsilon^{\gamma+1},
$$

where $\left.\eta_{\gamma}=\varrho^{\gamma}(2 \varrho-\gamma-1)\right) 0$ and $\varrho$ is the unique solution of $\varrho(1+\tanh (\varrho))=\gamma+1$.

Remark 2. When $\gamma=0$, we get a famous inequality: $|z|-z \tan h(z / \epsilon) \leq \eta_{0} \epsilon$, where $\eta_{0} \approx 0.2758$. In this paper, since both the sliding mode and the controller contain $\tan h(\cdot)$ functions, the inequalities (12) of Lemma 2 play an important role.

The aim of this paper is (1) to propose an effective control method so that the error state e can be remained within a small neighborhood of zero; (2) to construct the disturbance observer so that $\mathbf{F}(\mathbf{x})+\mathbf{d}(t)+\Delta \mathbf{u}$ can be accurately estimated; (3) to design a new integral sliding variable such that the chatter phenomenon of controller can be eliminated.

\section{Sliding Mode Control Design and Stability Analysis}

3.1. Construction of Integral Sliding Mode and Disturbance Observer. In order to design a sliding mode robust controller to make the tracking error e stable, the integral sliding variable needs to be designed. In [25], an integral terminal sliding variable is designed as

$$
\mathbf{s}=\mathbf{e}+\int_{0}^{t} \mu_{1} \operatorname{Sign}^{\gamma_{1}}(\mathbf{e}) \mathrm{d} \tau
$$

where $\mathbf{s}=\left[s_{1}, s_{2}, \ldots, s_{n}\right]^{T} \in R^{n}, \operatorname{Sign}^{\gamma_{1}}(\mathbf{e})=\left[\left|e_{1}\right|^{\gamma_{1}} \operatorname{sign}\left(e_{1}\right)\right.$, $\left.\left|e_{2}\right|^{\gamma_{1}} \operatorname{sign}\left(e_{2}\right), \ldots,\left|e_{n}\right|^{\gamma_{1}} \operatorname{sign}\left(e_{n}\right)\right]^{T}, \gamma_{1} \in[0,1)$, and $\mu_{1}$ is a positive parameter. By using the traditional sliding mode control method, the occurrence of chatter phenomenon is mainly due to the hysteresis of control switching. To avoid the chatter phenomenon, we modify the sliding surface (13) as follows:

$$
\mathbf{s}=\mathbf{e}+\int_{0}^{t}\left(\mu_{1} \operatorname{Tanh}_{\epsilon_{1}}^{\gamma_{1}}(\mathbf{e})+\mu_{2} \operatorname{Tanh}_{\epsilon_{1}}^{0}(\mathbf{e})\right) \mathrm{d} \tau,
$$

where $\operatorname{Tanh}_{\epsilon_{1}}^{\gamma_{1}}(\mathbf{e})=\left[\left|e_{1}\right|^{\gamma_{1}} \tan h\left(e_{1} / \epsilon_{1}\right),\left|e_{2}\right|^{\gamma_{1}} \tanh \left(e_{2} / \epsilon_{1}\right)\right.$, $\left.\ldots,\left|e_{n}\right|^{\gamma_{1}} \tanh \left(e_{n} / \epsilon_{1}\right)\right]^{T}, \operatorname{Tanh}_{\epsilon_{1}}^{0}(\mathbf{e})=\left[\tanh \left(e_{1} / \epsilon_{1}\right), \tanh \left(e_{2} /\right.\right.$ $\left.\left.\epsilon_{1}\right), \ldots, \tan h\left(e_{n} / \epsilon_{1}\right)\right]^{T}, \mu_{2}$ is the designed positive parameter and $\epsilon_{1}$ is a small positive constant, and $\gamma_{1}$ and $\mu_{1}$ are the same as in (13).

Remark 3. In general, the aim of using the integral sliding variable $\mathbf{s}$ in (13) is to improve its control performance and eliminate the reaching phase under the nominal control law. Different from the former, based on the dynamic system of integral sliding variable $s$ in (14), this paper is to explore the ultimate boundary of the tracking error $\mathbf{e}$.

According to (10) and (14), the dynamic system of $\mathbf{s}$ is obtained as follows:

$$
\begin{aligned}
\dot{\mathbf{s}}= & \mathbf{L}^{-1} \widetilde{\theta}_{\mathbf{F}}^{T} \varphi_{\mathbf{F}}(\mathbf{x})+\mathbf{L}^{-1} \widehat{\theta}_{\mathbf{F}}^{T} \varphi_{\mathbf{F}}(\mathbf{x})+\mathbf{u}+\mathbf{D}-\dot{\mathbf{x}}_{\mathbf{d}} \\
& +\mu_{1} \operatorname{Tanh}_{\epsilon_{1}}^{\gamma_{1}}(\mathbf{e})+\mu_{2} \operatorname{Tanh}_{\epsilon_{1}}^{0}(\mathbf{e}) .
\end{aligned}
$$

In order to show the performance of RBFNNs, defining $\widehat{\mathbf{s}}=\left[\widehat{\boldsymbol{s}}_{1}, \widehat{\boldsymbol{s}}_{2}, \ldots, \widehat{\boldsymbol{s}}_{n}\right]^{T}$ as the estimation of $\mathbf{s}$, we have

$$
\begin{aligned}
\dot{\widehat{\mathbf{s}}}= & -\mu_{3} \widetilde{\mathbf{s}}+\mathbf{L}^{-1} \hat{\theta}_{\mathbf{F}}^{T} \varphi_{\mathbf{F}}(\mathbf{x})+\mathbf{u}+\widehat{\mathbf{D}}-\dot{\mathbf{x}}_{\mathbf{d}}+\mu_{1} \operatorname{Tanh}_{\epsilon_{1}}^{\gamma_{1}}(\mathbf{e}) \\
& +\mu_{2} \operatorname{Tanh}_{\epsilon_{1}}^{0}(\mathbf{e}),
\end{aligned}
$$

where $\widetilde{\mathbf{s}}=\widehat{\mathbf{s}}-\mathbf{s}, \mu_{3}$ is the positive design constant, and $\widehat{\mathbf{D}}=$ $\left[\widehat{D}_{1}, \widehat{D}_{2}, \ldots, \widehat{D}_{n}\right]^{T}$ is the estimation of $\mathbf{D}$. Furthermore, the derivative of $\widetilde{\mathbf{s}}$ is obtained as

$$
\dot{\tilde{\mathbf{s}}}=-\mu_{3} \widetilde{\mathbf{s}}-\mathbf{L}^{-1} \tilde{\theta}_{\mathbf{F}}^{T} \varphi_{\mathbf{F}}(\mathbf{x})-\widetilde{\mathbf{D}}
$$


Now, the disturbance observer $\widehat{\mathbf{D}}$ is constructed as

$$
\left\{\begin{array}{l}
\widehat{\mathbf{D}}=\mu_{4}(\mathbf{s}-\mathbf{w}) \\
\dot{\mathbf{w}}=\mathbf{L}^{-1} \widehat{\theta}_{\mathbf{F}}^{T} \varphi_{\mathbf{F}}(\mathbf{x})+\mathbf{u}+\widehat{\mathbf{D}}-\dot{\mathbf{x}}_{\mathbf{d}}+\mu_{1} \operatorname{Tanh}_{\epsilon_{1}}^{\gamma_{1}}(\mathbf{e})+\mu_{2} \operatorname{Tanh}_{\epsilon_{1}}^{0}(\mathbf{e})+\mu_{4}^{-1}\left(-\mathbf{s}+\mu_{5} \widetilde{\mathbf{s}}\right)
\end{array}\right.
$$

where $\mathbf{w}=\left[w_{1}, w_{2}, \ldots, w_{n}\right]^{T} \in R^{n}$ is an auxiliary variable vector and $\mu_{4}$ and $\mu_{5}$ are positive design parameters.

The derivative of $\widehat{\mathbf{D}}$ can be written as

$$
\dot{\hat{\mathbf{D}}}=\mu_{4}(\dot{\mathbf{s}}-\dot{\mathbf{w}})=\mu_{4}\left(\mathbf{L}^{-1} \tilde{\theta}_{\mathbf{F}}^{T} \varphi_{\mathbf{F}}(\mathbf{x})+\widetilde{\mathbf{D}}\right)+\mathbf{s}-\mu_{5} \widetilde{\mathbf{s}}
$$

where $\widetilde{\mathbf{D}}=\mathbf{D}-\widehat{\mathbf{D}}=\left[\widetilde{D}_{1}, \widetilde{D}_{2}, \ldots, \widetilde{D}_{n}\right]^{T}$, and then, we obtain

$$
\dot{\widetilde{\mathbf{D}}}=\dot{\mathbf{D}}-\mathbf{s}+\mu_{5} \widetilde{\mathbf{s}}-\mu_{4}\left(\mathbf{L}^{-1} \widetilde{\theta}_{\mathbf{F}}^{T} \varphi_{\mathbf{F}}(\mathbf{x})+\widetilde{\mathbf{D}}\right)
$$

3.2. Main Results. Now, the controller $\mathbf{u}(t)$ is designed as

$$
\begin{aligned}
\mathbf{u}= & -\mu_{6} \mathbf{s}-\mu_{7} \operatorname{Tanh}_{\epsilon_{2}}^{\gamma_{2}}(\mathbf{s})-\mathbf{L}^{-1} \widehat{\theta}_{\mathbf{F}}^{T} \varphi_{\mathbf{F}}(\mathbf{x})-\widehat{\mathbf{D}}+\dot{\mathbf{x}}_{\mathbf{d}} \\
& -\mu_{1} \operatorname{Tanh}_{\epsilon_{1}}^{\gamma_{1}}(\mathbf{e})-\mu_{2} \operatorname{Tanh}_{\epsilon_{1}}^{0}(\mathbf{e}),
\end{aligned}
$$

and the parameter adaptive laws are proposed as

$$
\dot{\hat{\theta}}_{\bar{f}_{i}}=\eta_{\bar{f}_{i}}\left[l_{i}^{-1} s_{i} \varphi_{\bar{f}_{i}}(\mathbf{x})-\mu_{5} l_{i}^{-1} \widetilde{s}_{i} \varphi_{\bar{f}_{i}}(\mathbf{x})-\delta_{1} \widehat{\theta}_{\bar{f}_{i}}\right],
$$

where $\operatorname{Tanh}_{\epsilon_{2}}^{\gamma_{2}}(\mathbf{s})=\left[\left|s_{1}\right|^{\gamma_{2}} \quad \tan h\left(s_{1} / \epsilon_{2}\right),\left|s_{2}\right|^{\gamma_{2}} \tan h\left(s_{2} / \epsilon_{2}\right)\right.$, $\left.\ldots,\left|s_{n}\right|^{\gamma_{2}} \tan h\left(s_{n} / \epsilon_{2}\right)\right]^{T}, \delta_{1}, \mu_{6}, \mu_{7}$, and $\eta_{\bar{f}_{i}}$ are the design parameters, $i=1,2, \ldots, n$, and $\epsilon_{2}$ is a small positive constant. We give the main result as follows.

Theorem 1. For the error system (10) under Assumptions 1-4 and the disturbance observer (18), the controller (21) and parameter adaptive laws (22) guarantee that all the closedloop system signals in (23) are uniformly ultimately bounded.
Proof. Consider the Lyapunov function as

$$
V=V_{1}+V_{2}+V_{3}+V_{4} \text {, }
$$

where

$$
\begin{aligned}
& V_{1}=\frac{1}{2} \mathbf{s}^{T} \mathbf{s}=\frac{1}{2}\|\mathbf{s}\|^{2}=\sum_{i=1}^{n} \frac{1}{2} s_{i}^{2}, \\
& V_{2}=\frac{1}{2} \widetilde{\mathbf{D}}^{T} \widetilde{\mathbf{D}}=\frac{1}{2}\|\widetilde{\mathbf{D}}\|^{2}=\sum_{i=1}^{n} \frac{1}{2} \widetilde{D}_{i}^{2}, \\
& V_{3}=\frac{\mu_{5}}{2} \widetilde{\mathbf{s}}^{T} \widetilde{\mathbf{s}}=\frac{\mu_{5}}{2}\|\widetilde{\mathbf{s}}\|^{2}=\sum_{i=1}^{n} \frac{\mu_{5}}{2} \widetilde{s}_{i}^{2}, \\
& V_{4}=\sum_{i=1}^{n} \frac{1}{2 \eta_{\bar{f}_{i}}}\left\|\widetilde{\theta}_{\bar{f}_{i}}\right\|^{2}=\sum_{i=1}^{n} \frac{1}{2 \eta_{\bar{f}_{i}}} \widetilde{\theta}_{\bar{f}_{i}}^{T} \widetilde{\theta}_{\bar{f}_{i}} .
\end{aligned}
$$

Substituting (21) into (15), the derivative of $V_{1}$ can be obtained as

$$
\begin{aligned}
\dot{V}_{1} & =\mathbf{s}^{T} \dot{\mathbf{s}}=-\mu_{6} \mathbf{s}^{T} \mathbf{s}-\mu_{7} \mathbf{s}^{T} \mathbf{T a n h}_{\epsilon_{2}}^{\gamma_{2}}(\mathbf{s})+\mathbf{s}^{T} \widetilde{\mathbf{D}}+\mathbf{s}^{T} \mathbf{L}^{-1} \widetilde{\theta}_{\mathbf{F}}^{T} \varphi_{\mathbf{F}}(\mathbf{x}) \\
& =-\mu_{6} \mathbf{s}^{T} \mathbf{s}-\sum_{i=1}^{n} \mu_{7} s_{i}\left|s_{i}\right|^{\gamma_{2}} \tanh \left(\frac{s_{i}}{\epsilon_{2}}\right)+\mathbf{s}^{T} \widetilde{\mathbf{D}}+\sum_{i=1}^{n} s_{i} l_{i}^{-1} \widetilde{\theta}_{f_{i}}^{T} \varphi_{\bar{f}}(\mathbf{x}) .
\end{aligned}
$$

From (20), the derivative of $V_{2}$ yields

$$
\dot{V}_{2}=\widetilde{\mathbf{D}}^{T} \dot{\widetilde{\mathbf{D}}}=\widetilde{\mathbf{D}}^{T} \dot{\mathbf{D}}-\mu_{4} \widetilde{\mathbf{D}}^{T} \widetilde{\mathbf{D}}-\widetilde{\mathbf{D}}^{T} \mathbf{s}+\mu_{5} \widetilde{\mathbf{D}}^{T} \widetilde{\mathbf{s}}-\sum_{i=1}^{n} \mu_{4} \widetilde{D}_{i} l_{i}^{-1} \widetilde{\theta}_{f_{i}}^{T} \varphi_{\bar{f}_{i}}(\mathbf{x})
$$

From (17), the derivative of $V_{3}$ yields

From (22), the derivative of $V_{4}$ yields

$$
\dot{V}_{3}=\mu_{5} \widetilde{\mathbf{s}}^{T} \dot{\widetilde{\mathbf{s}}}=-\mu_{3} \mu_{5} \widetilde{\mathbf{s}}^{T} \widetilde{\mathbf{s}}-\mu_{5} \widetilde{\mathbf{s}}^{T} \widetilde{\mathbf{D}}-\sum_{i=1}^{n} \mu_{5} l_{i}^{-1} \widetilde{s}_{i} \widetilde{\theta}_{\bar{f}_{i}}^{T} \varphi_{\bar{f}_{i}}(\mathbf{x}) \text {. }
$$

$$
\dot{V}_{4}=\sum_{i=1}^{n} \frac{1}{\eta_{\bar{f}_{i}}} \widetilde{\theta}_{\bar{f}_{i}}^{T} \dot{\tilde{\theta}}_{\bar{f}_{i}}=\sum_{i=1}^{n}\left[-l_{i}^{-1} s_{i} \widetilde{\theta}_{\bar{f}_{i}}^{T} \varphi_{\bar{f}_{i}}(\mathbf{x})+\mu_{5} l_{i}^{-1} \widetilde{s}_{i} \widetilde{\theta}_{\bar{f}_{i}}^{T} \varphi_{\bar{f}_{i}}(\mathbf{x})+\delta_{1} \widetilde{\theta}_{\bar{f}_{i}}^{T} \widehat{\theta}_{\bar{f}_{i}}\right]
$$

Using (25), (26), (27) and (28), we have 


$$
\dot{V}=-\mu_{6} \mathbf{s}^{T} \mathbf{s}-\sum_{i=1}^{n} \mu_{7} s_{i}\left|s_{i}\right|^{\gamma_{2}} \tan h\left(\frac{s_{i}}{\epsilon_{2}}\right)+\widetilde{\mathbf{D}}^{T} \dot{\mathbf{D}}-\mu_{4} \widetilde{\mathbf{D}}^{T} \widetilde{\mathbf{D}}-\sum_{i=1}^{n} \mu_{4} \widetilde{D}_{i} l_{i}^{-1} \widetilde{\theta}_{f_{i}}^{T} \varphi_{\bar{f}_{i}}(\mathbf{x})-\mu_{3} \mu_{5} \widetilde{\mathbf{s}}^{T} \widetilde{\mathbf{s}}+\sum_{i=1}^{n} \delta_{1} \widetilde{\theta}_{\bar{f}_{i}}^{T} \widehat{\theta}_{\bar{f}_{i}} .
$$

Since the following inequalities hold,

$$
\begin{aligned}
\widetilde{\mathbf{D}}^{T} \dot{\mathbf{D}} \leq \frac{\|\widetilde{\mathbf{D}}\|^{2}}{2}+\frac{\|\dot{\mathbf{D}}\|^{2}}{2} \leq \frac{\|\widetilde{\mathbf{D}}\|^{2}}{2}+\frac{D^{* 2}}{2}, \\
-\mu_{4} l_{i}^{-1} \widetilde{D}_{i} \widetilde{\theta}_{\bar{f}_{i}}^{T} \varphi_{\bar{f}_{i}}(\mathbf{x}) \leq \frac{\mu_{4} p^{* 2} \widetilde{D}_{i}^{2}}{2 l_{\min }}+\frac{\mu_{4}\left\|\widetilde{\theta}_{\bar{f}_{i}}\right\|^{2}}{2 l_{\min }}, \\
\delta_{1} \widetilde{\theta}_{\bar{f}_{i}}^{T} \widehat{\theta}_{\bar{f}_{i}} \leq-\frac{\delta_{1}}{2}\left\|\widetilde{\theta}_{\bar{f}_{i}}\right\|^{2}+\frac{\delta_{1}}{2}\left\|\theta_{\bar{f}_{i}}^{*}\right\|^{2},
\end{aligned}
$$

where $l_{\min }=\min \left\{l_{1}, l_{2}, \ldots, l_{n}\right\}$.

By using Lemma 1, one has

$$
-\sum_{i=1}^{n} \mu_{7} s_{i}\left|s_{i}\right|^{\gamma_{2}} \tanh \left(\frac{s_{i}}{\epsilon_{2}}\right) \leq-\sum_{i=1}^{n} \mu_{7}\left|s_{i}\right|^{\gamma_{2}+1}+\mu_{7} n \eta_{\gamma_{2}} \epsilon_{2}^{\gamma_{2}+1}
$$

Since $0<\gamma_{2}+1<2$, the following inequality holds by using Lemma 2:

$$
\|\mathbf{s}\|=\left(\sum_{i=1}^{n}\left|s_{i}\right|^{2}\right)^{(1 / 2)} \leq\left(\sum_{i=1}^{n}\left|s_{i}\right|^{\gamma_{2}+1}\right)^{\left(1 / \gamma_{2}+1\right)} .
$$

Substituting (32) into (31) yields

$$
-\sum_{i=1}^{n} \mu_{7} s_{i}\left|s_{i}\right|^{\gamma_{2}} \tanh \left(\frac{s_{i}}{\epsilon_{2}}\right) \leq-\mu_{7}\|\mathbf{s}\|^{\gamma_{2}+1}+\mu_{7} n \eta_{\gamma_{2}} \epsilon_{2}^{\gamma_{2}+1} \text {. }
$$

Substituting (30) and (33) into (29) results in

$$
\dot{V} \leq-\mu_{6}\|\mathbf{s}\|^{2}-\mu_{7}\|\mathbf{s}\|^{\gamma_{2}+1}-\left(\mu_{4}-\frac{1}{2}-\frac{\mu_{4}}{2 k_{\text {min }}}-\frac{\mu_{4}}{2 l_{\text {min }}}\right)\|\tilde{\mathbf{D}}\|^{2}-\mu_{3} \mu_{5}\|\widetilde{\mathbf{s}}\|^{2}-\sum_{i=1}^{n}\left(\frac{\delta_{1}}{2}-\frac{\mu_{4}}{2 l_{\text {min }}}\right)\left\|\tilde{\theta}_{\bar{f}_{i}}\right\|^{2}+r_{0},
$$

where $r_{0}=\left(D^{* 2} / 2\right)+\mu_{7} n \eta_{\gamma_{2}} \epsilon_{2}^{\gamma_{2}+1}+\left(\delta_{1}^{2} / 2\right) \sum_{i=1}^{n}\left\|\theta_{f_{i}}^{*}\right\|^{2}$. By choosing parameters $\mu_{4}, l_{\text {min }}$ and $\delta_{1}$ satisfy

$$
\begin{aligned}
& \lambda_{D}=\mu_{4}-\frac{1}{2}-\frac{\mu_{4}}{2 k_{\text {min }}}-\frac{\mu_{4}}{2 l_{\text {min }}}>0, \\
& \lambda_{\theta_{\mathrm{F}}}=\frac{\delta_{1}}{2}-\frac{\mu_{4}}{2 l_{\text {min }}}>0,
\end{aligned}
$$
and let $r_{\min }=\min \left\{\left(\mu_{6} / 2\right),\left(\lambda_{D} / 2\right),\left(\mu_{3} / 2\right), \eta_{\bar{f}_{1}} \lambda_{\theta_{\mathbf{F}}}, \ldots, \eta_{\bar{f}_{n}} \lambda_{\theta_{\mathbf{F}}}\right\}$.
It can be obtained as

$$
\dot{V} \leq-r_{\min } V+r_{0}
$$

From (36), we know

$$
V \leq \frac{r_{0}}{r_{\min }}+V(0) e^{-r_{\min } t}
$$

Obviously, it can conclude that all signals in (23) are ultimately uniformly bounded. The proof is completed.

Theorem 2. Based on Theorem 1 and the dynamic system of the integral sliding mode variable itself, the ultimate bound of e is estimated as $\|\mathbf{e}\| \leq\left(\epsilon_{0} / \mu_{1}(1-\varsigma)\right)^{\left(1 / \gamma_{1}+1\right)}$.

Proof. Theorem 1 shows that the designed sliding mode controller (21) guarantees that all signals in (23) are bounded. Now, we further study the convergence of the tracking error e. According to (14), (15), and (21), we have

$$
\dot{\mathbf{e}}=\dot{\mathbf{s}}-\mu_{1} \operatorname{Tanh}_{\epsilon_{1}}^{\gamma_{1}}(\mathbf{e})-\mu_{2} \operatorname{Tanh}_{\epsilon_{1}}^{0}(\mathbf{e})=\psi\left(\mathbf{s}, \widetilde{\mathbf{D}}, \widetilde{\theta}_{\bar{f}_{1}}, \ldots, \widetilde{\theta}_{\bar{f}_{n}}\right)-\mu_{1} \operatorname{Tanh}_{\epsilon_{1}}^{\gamma_{1}}(\mathbf{e})-\mu_{2} \operatorname{Tanh}_{\epsilon_{1}}^{0}(\mathbf{e})
$$

where $\psi\left(\mathbf{s}, \widetilde{\mathbf{D}}, \widetilde{\theta}_{\bar{f}_{1}}, \ldots, \quad \widetilde{\theta}_{\bar{f}_{n}}\right)=\mathbf{L}^{-1} \widetilde{\theta}_{\mathbf{F}}^{T} \varphi_{\mathbf{F}}(\mathbf{x})+\widetilde{\mathbf{D}}-\mu_{6} \mathbf{s}-$ $\mu_{7} \operatorname{Tanh}_{\epsilon_{2}}^{\gamma_{2}}(\mathbf{s})$. Through the conclusion of Theorem 1 , there exists a positive constant $₫$ such that

$$
\left\|\psi\left(\mathbf{s}, \widetilde{\mathbf{D}}, \widetilde{\theta}_{\bar{f}_{1}}, \ldots, \tilde{\theta}_{\bar{f}_{n}}\right)\right\| \leq \varpi .
$$

Consider the following Lyapunov function:

$$
\bar{V}=\frac{1}{2} \mathbf{e}^{T} \mathbf{e} .
$$

The derivative of $\bar{V}$ is calculated as 


$$
\dot{\bar{V}}=\mathbf{e}^{T} \psi\left(\mathbf{s}, \widetilde{\mathbf{D}}, \widetilde{\theta}_{\bar{f}_{1}}, \ldots, \widetilde{\theta}_{\bar{f}_{n}}\right)-\mu_{1} \mathbf{e}^{T} \operatorname{Tanh}_{\epsilon_{1}}^{\gamma_{1}}(\mathbf{e})-\mu_{2} \mathbf{e}^{T} \operatorname{Tanh}_{\epsilon_{1}}^{0}(\mathbf{e}) \leq \Phi\|\mathbf{e}\|-\sum_{i=1}^{n} \mu_{1} e_{i}\left|e_{i}\right|^{\gamma_{1}} \tan h\left(\frac{e_{i}}{\epsilon_{1}}\right)-\sum_{i=1}^{n} \mu_{2} e_{i} \tan h\left(\frac{e_{i}}{\epsilon_{1}}\right) .
$$

By using Lemma 1 and 2, the following inequalities hold:

$$
\begin{gathered}
-\sum_{i=1}^{n} \mu_{1} e_{i}\left|e_{i}\right|^{\gamma_{1}} \tanh \left(\frac{e_{i}}{\epsilon_{1}}\right) \leq-\sum_{i=1}^{n} \mu_{1}\left|e_{i}\right|^{\gamma_{1}+1}+\mu_{1} n \eta_{\gamma_{1}} \epsilon_{1}^{\gamma_{1}+1} \leq-\mu_{1}\|\mathbf{e}\|^{\gamma+1}+\mu_{1} n \eta_{\gamma_{1}} \epsilon_{1}^{\gamma_{1}+1}, \\
-\sum_{i=1}^{n} \mu_{2} e_{i} \tanh \left(\frac{e_{i}}{\epsilon_{1}}\right) \leq-\sum_{i=1}^{n} \mu_{2}\left|e_{i}\right|+\mu_{2} n \eta_{\gamma_{0}} \epsilon_{1} \leq-\mu_{2}\|\mathbf{e}\|+\mu_{2} n \eta_{\gamma_{0}} \varepsilon_{1} .
\end{gathered}
$$

Substituting (42) and (43) into (41), we have

$$
\dot{\bar{V}} \leq-\left(\mu_{2}-\omega\right)\|\mathbf{e}\|-\mu_{1}\|\mathbf{e}\|^{\gamma_{1}+1}+\epsilon_{0},
$$

where $\epsilon_{0}=\mu_{1} n \eta_{\gamma_{1}} \epsilon_{1}^{\gamma_{1}+1}+\mu_{2} n \eta_{\gamma_{0}} \epsilon_{1}$. Selecting $\mu_{2}$ satisfies $\epsilon_{0}=\mu_{1} n \eta_{\gamma_{1}} \epsilon_{1}^{\gamma_{1}+1}+\mu_{2} n \eta_{\gamma_{0}} \epsilon_{1}$, and define the compact set as

$$
\Omega_{\mathbf{e}}=\left\{\mathbf{e} \in R^{n} \mid \mu_{1}\|\mathbf{e}\|^{\gamma_{1}+1} \leq \frac{\epsilon_{0}}{1-\varsigma}\right\}
$$

where $\varsigma \in(0,1)$ is any number. If $\mathbf{e} \notin \Omega_{\mathbf{e}}$, then

$$
\dot{\bar{V}} \leq-\mu_{1}\|\mathbf{e}\|^{\gamma_{1}+1}+\epsilon_{0}<-\mu_{1} \varsigma\|\mathbf{e}\|^{\gamma_{1}+1}=-2^{\left(\gamma_{1}+1 / 2\right)} \mu_{1} \varsigma \bar{V}^{\left(\gamma_{1}+1 / 2\right)} \text {. }
$$

Obviously, $\bar{V}$ decreases monotonically outside the set $\Omega_{\mathrm{e}}$ until it enters the minimal level set of $\bar{V}$ containing $\Omega_{\mathbf{e}}$.

Remark 4. Because parameter $\epsilon_{1}$ can be selected as a small positive constant, which means that parameter $\epsilon_{0}$ is also very small, $\Omega_{\mathrm{e}}$ is a very small neighborhood.

\section{Example}

In this section, the chaotic finance system [26] is introduced to demonstrate the effectiveness of the proposed control method (21) and the chaotic finance system is described as follows:

$$
\left\{\begin{array}{l}
\dot{x}_{1}=\underbrace{x_{3}-x_{1}\left(x_{2}-0.8\right)}_{f_{1}(\mathbf{x})}+d_{1}(t)+\operatorname{sat}\left(u_{1}\right), \\
\dot{x}_{2}=\underbrace{1-x_{1}^{2}-0.2 x_{2}}_{f_{2}(\mathbf{x})}+d_{2}(t)+\operatorname{sat}\left(u_{2}\right), \\
\dot{x}_{3}=\underbrace{-1.9 x_{3}-x_{1}}_{f_{3}(\mathbf{x})}+d_{3}(t)+\operatorname{sat}\left(u_{3}\right),
\end{array}\right.
$$

where $d_{1}(t)=2.5 \sin (t), d_{2}(t)=2.0 \cos (2 t)$, and $d_{3}(t)=$ $2.5 \sin (3 t)$. The reference signal $\mathbf{x}_{\mathbf{d}}=[\sin (t), \sin (t), \sin (t)]^{T}$. Here, initial values of $\mathbf{x}, \widetilde{\mathbf{s}}$, and $\mathbf{w}$ are chose as $\mathbf{x}(0)=[2,-1,-2.5]^{T}, \quad \widehat{\mathbf{s}}(0)=[1.2,1.2,1.2]^{T}$, and $\mathbf{w}(0)=$ $[-2,2,3]^{T}$, and the parameters are chose as $u_{i}^{*}=10, l_{i}=5$, $\eta_{f_{i}}=50, \quad \mu_{1}=\mu_{2}=5, \mu_{3}=\mu_{5}=\mu_{7}=3, \mu_{4}=15, \mu_{6}=4$, $\epsilon_{1}=\epsilon_{2}=0.0005, \varsigma=(1 / 7), \delta_{1}=0.01$, and $i=1,2,3$. The fuzzy membership functions are selected as

$$
\varphi_{f}(\zeta)=\exp \left[-\frac{1}{2}\left(\frac{\zeta+7.5-2.5 k}{1.2}\right)^{2}\right]
$$

where $k=1,2,3,4,5 ; \zeta=x_{1}, x_{2}, x_{3}$.

According to Theorem 2, the ultimate bound of the tracking error e can be estimated in the interval $(-2.4 \times$ $\left.10^{-3}, 2.4 \times 10^{-3}\right)$ when $\gamma_{1}=(1 / 5)$. Now, we give the traditional integral sliding mode control method (TISMC) as follows:

$$
\begin{aligned}
\mathbf{s} & =\mathbf{e}+\int_{0}^{t} \mu_{1} \operatorname{Sign}^{\gamma_{1}}(\mathbf{e}) \mathrm{d} \tau \\
\mathbf{u} & =-\mu_{6} \mathbf{s}-\mu_{7} \operatorname{sign}^{\gamma_{2}}(\mathbf{s})-\widehat{\vartheta}_{\overline{\mathbf{F}}}^{T} \varphi_{\overline{\mathbf{F}}}(\mathbf{x})+\dot{\mathbf{x}}_{\mathbf{d}}-\mu_{1} \operatorname{Sign}^{\gamma_{1}}(\mathbf{e}), \\
\dot{\widehat{\vartheta}}_{\bar{F}_{i}} & =50\left[s_{i} \varphi_{\bar{F}}(\mathbf{x})-0.01 \widehat{\vartheta}_{\bar{F}_{i}}\right]
\end{aligned}
$$

where $\widehat{\vartheta}_{\overline{\mathbf{F}}}^{T} \varphi_{\overline{\mathbf{F}}}(\mathbf{x})=\left[\widehat{\vartheta}_{\bar{F}_{1}}^{T} \varphi_{\bar{F}}(\mathbf{x}), \widehat{\vartheta}_{\bar{F}_{2}}^{T} \varphi_{\bar{F}}(\mathbf{x}), \widehat{\vartheta}_{\bar{F}_{3}}^{T} \varphi_{\bar{F}}(\mathbf{x})\right]^{T}$ is the estimation of $F(\mathbf{x})+\Delta \mathbf{u}+\mathbf{d}(t)$. Obviously, the approximation range of e cannot be obtained directly from TISMC (49). In the following, TISMC (49) is discussed in three cases.

(i) Case 1: $\gamma_{1}=(1 / 5), \gamma_{2}=(1 / 5)$.

Figures 1 and 2 show the simulation results for case 1 . It can be seen that the tracking error quickly approaches zero, but the fluctuation range of the error $\mathbf{e}$ exceeds $\left(-2.4 \times 10^{-3}, 2.4 \times 10^{-3}\right)$, and both the error state $\mathbf{e}=\left[e_{1}, e_{2}, e_{3}\right]^{T}$ and the saturation control input $\operatorname{sat}(\mathbf{u})=\left[\operatorname{sat}\left(u_{1}\right) \text {, sat }\left(u_{2}\right) \operatorname{sat}\left(u_{3}\right)\right]^{T}$ appear as chattering phenomenon.

Case 2: $\gamma_{1}=(1 / 8), \gamma_{2}=(1 / 5)$.

Figures 3 and 4 show the simulation results for case 2 . It can be seen from Figure 3 that the convergence speed of the error $\mathbf{e}$ is the same as that of case 1 , and the fluctuation range of the error $\mathbf{e}$ is limited to the interval $\left(-2.4 \times 10^{-3}, 2.4 \times 10^{-3}\right)$. However, Figures 3 and 4 also reflect the chattering phenomenon of the error state $\mathbf{e}$ and saturation control input sat $(\mathbf{u})$ is more severe than that of case 1.

Case 3: $\gamma_{1}=(1 / 3), \gamma_{2}=(1 / 5)$.

Figures 5 and 6 show the simulation results for case 3 . Figure 6 shows that the chattering phenomenon of the saturation control input sat $(\mathbf{u})$ is reduced, but it is 


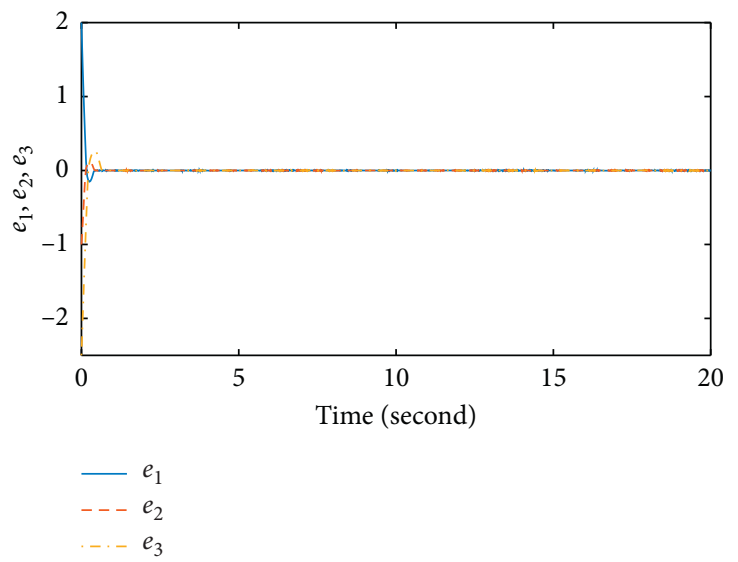

(a)

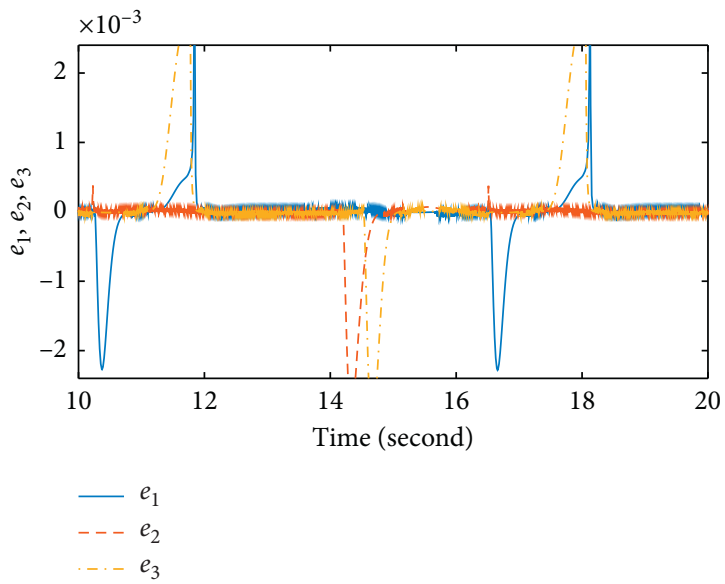

(b)

FIgURE 1: Case 1: the trajectories of tracking error $e_{1}, e_{2}, e_{3}$ by method (48) in time period: (a) [0, 20s]; (b) [10s, 20s].

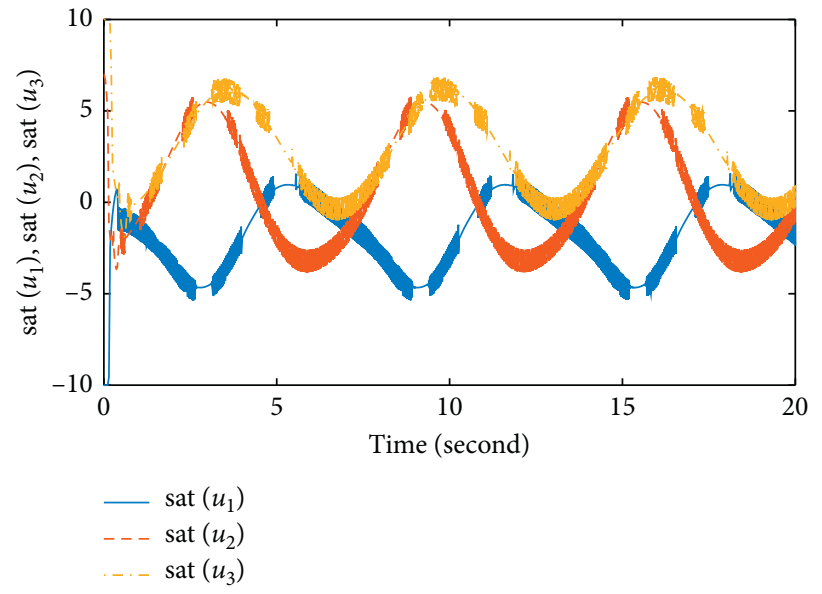

Figure 2: Case 1: the trajectories of $\operatorname{sat}\left(u_{1}\right)$, sat $\left(u_{2}\right)$, and sat $\left(u_{3}\right)$ by using method $(48)$.

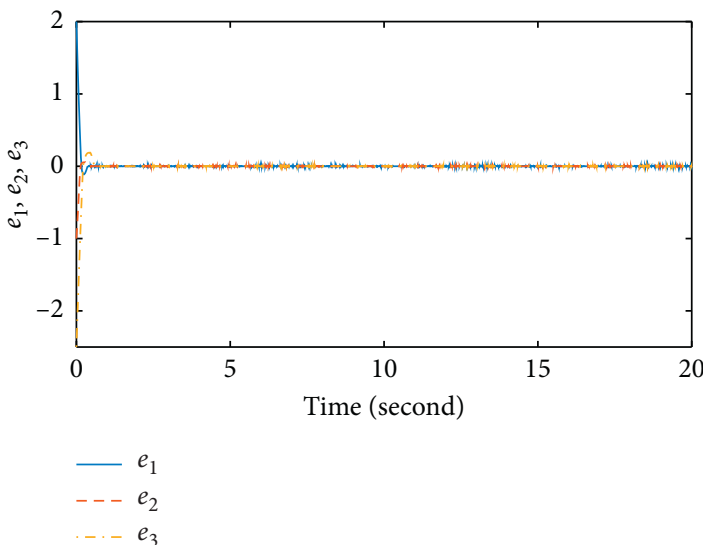

(a)

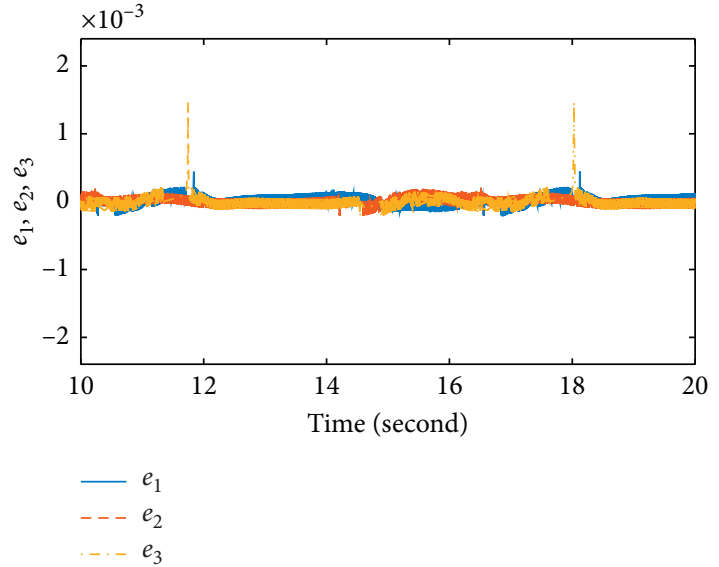

(b)

Figure 3: Case 2: the trajectories of tracking error $e_{1}, e_{2}, e_{3}$ by method (49) in time period: (a) [0, 20s]; (b) [10s, 20s]. 


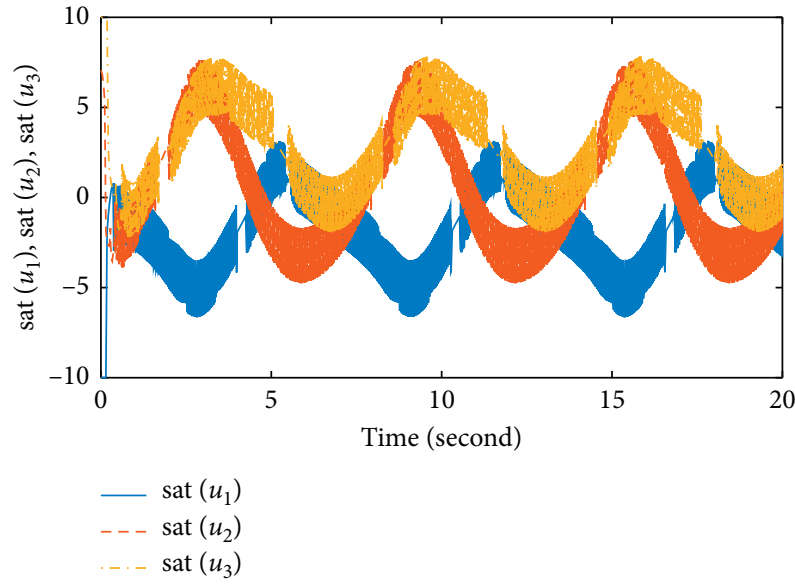

FIGURE 4: Case 2: the trajectories of $\operatorname{sat}\left(u_{1}\right)$, sat $\left(u_{2}\right)$, and sat $\left(u_{3}\right)$ by using method (49).
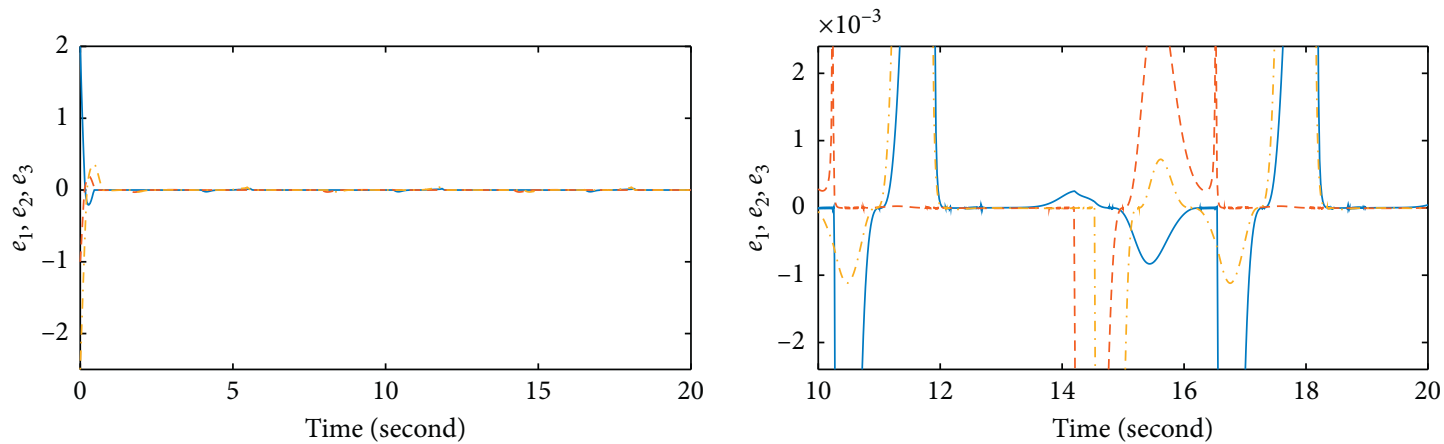

$$
\begin{aligned}
& -e_{1} \\
& --e_{2} \\
& -.-e_{3}
\end{aligned}
$$

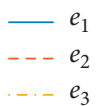

(a)

(b)

FIGURE 5: Case 3: the trajectories of tracking error $e_{1}, e_{2}, e_{3}$ by method (49) in time period: (a) [0, 20s]; (b) [10s, 20s].

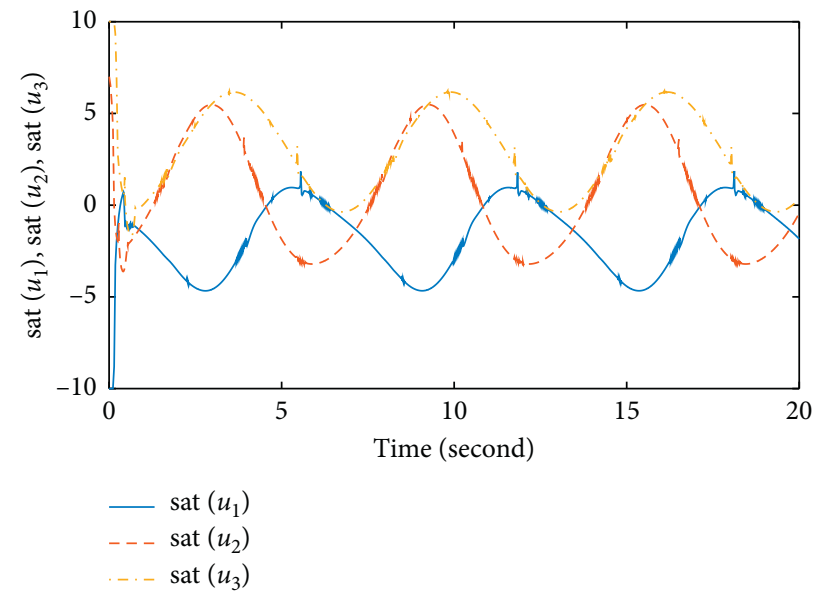

FIgURE 6: Case 3: the trajectories of $\operatorname{sat}\left(u_{1}\right)$, sat $\left(u_{2}\right)$, and sat $\left(u_{3}\right)$ by using method $(49)$. 


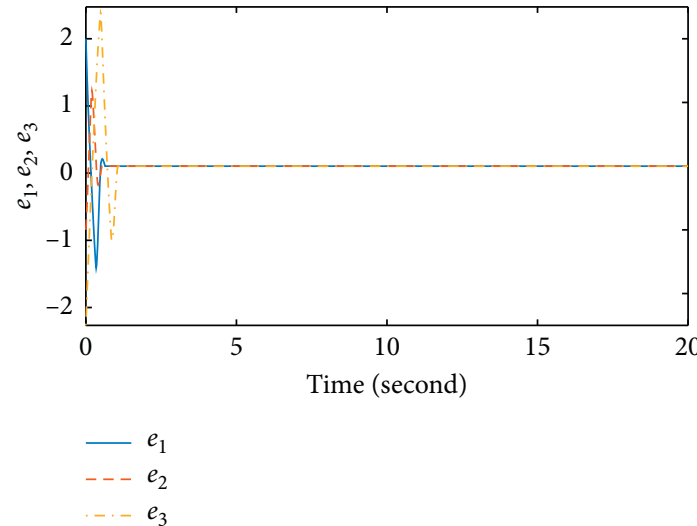

(a)

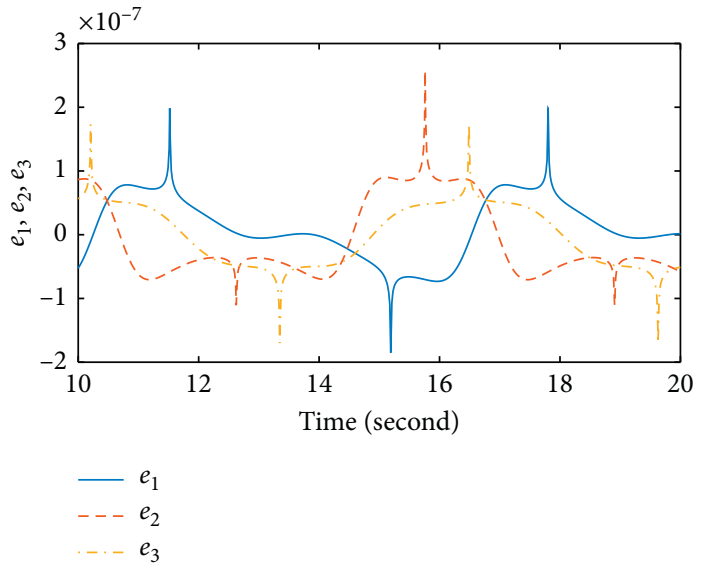

(b)

Figure 7: The trajectories of tracking error $e_{1}, e_{2}, e_{3}$ by method (21) in time period: (a) [0, 20 s]; (b) [10 s, $\left.20 \mathrm{~s}\right]$.

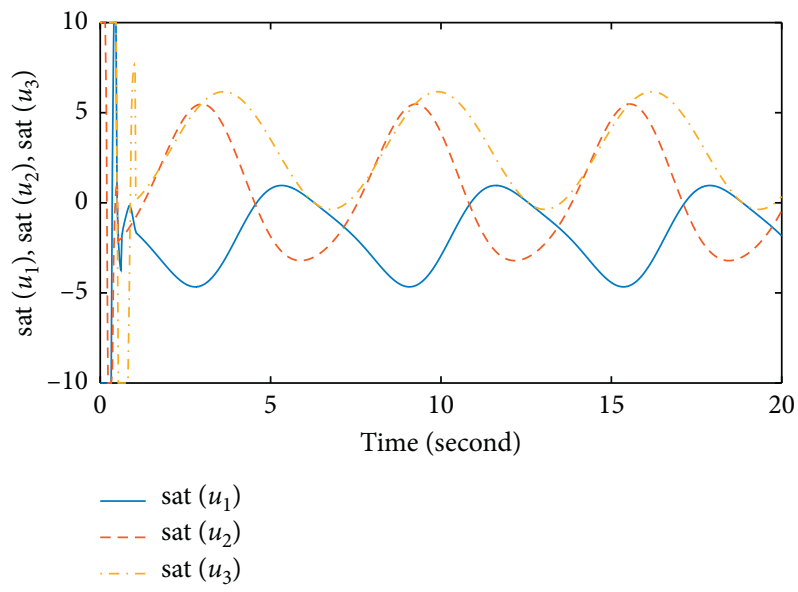

Figure 8 : The trajectories of sat $\left(u_{1}\right)$, sat $\left(u_{2}\right)$, and sat $\left(u_{3}\right)$ by using method $(21)$.

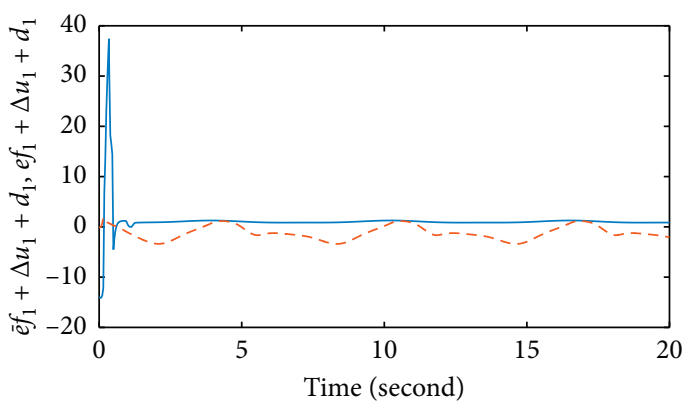

- $\bar{e} f_{1}+\Delta u_{1}+d_{1}$ by using method (20)

-.- ef $f_{1}+\Delta u_{1}+d_{1}$ by using method (47)

(a)

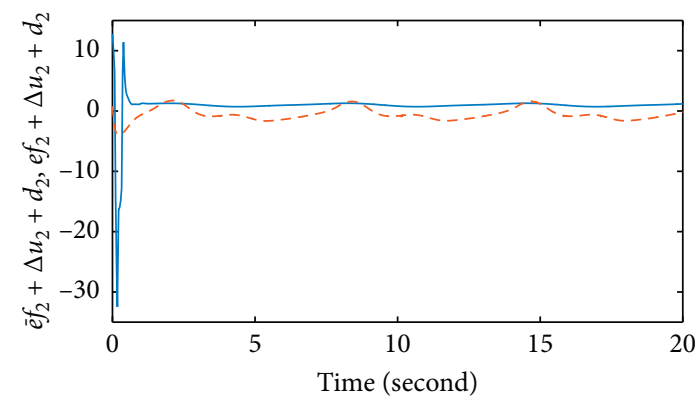

$-\bar{e} f_{2}+\Delta u_{2}+d_{2}$ by using method (20)
$--e f_{2}+\Delta u_{2}+d_{2}$ by using method (47)

(b)

FIgURE 9: Continued. 


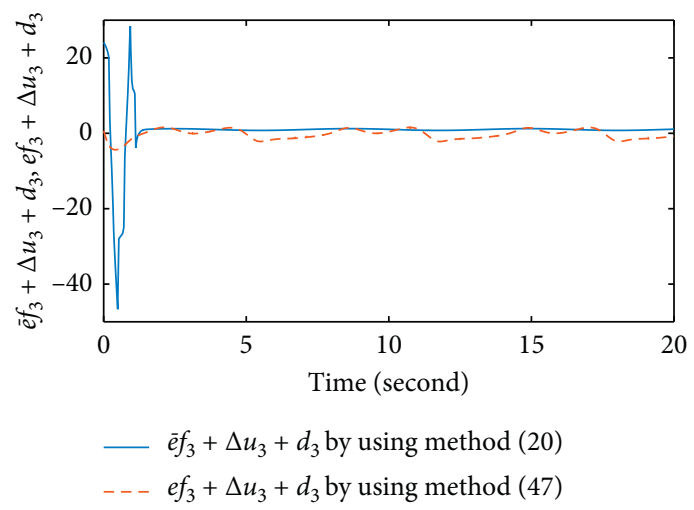

(c)

FIGURE 9: The trajectories of (a) $\bar{e}_{f_{1}+\Delta u_{1}+d_{1}}, e_{f_{1}+\Delta u_{1}+d_{1}}$; (b) $\bar{e}_{f_{2}+\Delta u_{2}+d_{2}}, e_{f_{2}+\Delta u_{2}+d_{2}}$; and (c) $\bar{e}_{f_{3}+\Delta u_{3}+d_{3}}, e_{f_{3}+\Delta u_{3}+d_{3}}$ by using methods (21) and (49).

found from Figure 5 that the effect of the error approaching zero is severely weakened compared to cases 1 and 2 .

The simulation results of cases $1-3$ show that the parameters $\gamma_{1}$ and $\gamma_{2}$ in control method (49) have a direct influence on the control effect of the error e. In other words, the smaller the fluctuation range of the error $\mathbf{e}$, the more violent the chattering phenomenon of the control input.

By using the proposed control method (21) in this paper, simulation results are shown in Figures 7 and 8 when $\gamma_{1}=\gamma_{2}=(1 / 5)$. It can be seen from Figure 7 that $\mathbf{e}$ is completely within the interval $\left(-2.4 \times 10^{-3}, 2.4 \times 10^{-3}\right)$, and it is found from Figure 8 that there is no chattering phenomenon in the saturation control input sat $(\mathbf{u})$. Let $\bar{e}_{f_{i}+\Delta u_{i}+d_{i}}=l_{i}^{-1} \widehat{\theta}_{\bar{f}_{i}}^{T} \varphi_{\bar{f}}$ $(\mathbf{x})+\widehat{D}_{i}-f_{i}(\mathbf{x})-\Delta u_{i}-d_{i}(t)$ and $e_{f_{i}+\Delta u_{i}+d_{i}}=\widehat{\vartheta}_{\bar{F}_{i}}^{T} \varphi_{\bar{F}}(\mathbf{x})-f_{i}$ $(\mathbf{x})-\Delta u_{i}-d_{i}(t)$ be the errors of system uncertainty by using methods (21) and (49), respectively. It can be seen from Figure 9 that the proposed control method (21) can better estimate system uncertainty than control method (49). Therefore, through simulation results, it is better to show that the proposed control method (21) has better control effect than method (49).

\section{Conclusion}

By using an integrated sliding mode control strategy and disturbance observer, this paper considers the tracking stability problem of a class of uncertain chaotic systems with saturation inputs. Firstly, the unknown function $\mathbf{F}(\mathbf{x})$ is estimated by using fuzzy logic systems, and the external disturbance $\mathbf{d}(t)$, the fuzzy estimation error $L^{-1} \varepsilon_{\mathrm{F}}(x)$, and the control error $\Delta \mathbf{u}=\operatorname{sat}(\mathbf{u})-\mathbf{u}$ are regarded as an unknown compound disturbance $\mathbf{D}$. Then, the integral sliding variable $\mathbf{s}$ is proposed, and the disturbance observer $\widehat{\mathbf{D}}$ is constructed by $\mathbf{s}$ and $\mathbf{D}$. The proposed control strategy $\mathbf{u}$ and parameter adaptive rules $\widehat{\theta}_{\bar{f}_{i}}$ can ensure that all signals of the closed-loop system are bounded, and the ultimate bound of the tracking error can be estimated. Simulation results show that the proposed method in this paper can effectively control the tracking error quickly and eliminate the chattering phenomenon. Meanwhile, the system uncertainty $\mathbf{F}(\mathbf{x})+\Delta \mathbf{u}+\mathbf{d}(t)$ can be effectively estimated.

\section{Data Availability}

All datasets generated for this study are included in the manuscript.

\section{Conflicts of Interest}

The authors declare that there are no conflicts of interest regarding the publication of this paper.

\section{References}

[1] O. Malley, J. Robert, E. Neubert, Michael, Fujiwara, and Masami, "Introduction to chaos: physics and mathematics of chaotic phenomena," in Siam Review, Taylor and FrancisMilton Park, UK, 2000.

[2] W. Al-Hussaibi, "Effect of filtering on the synchronization and performance of chaos-based secure communication over Rayleigh fading channel," Communications in Nonlinear Science and Numerical Simulation, vol. 26, no. 1-3, pp. 87-97, 2015.

[3] C. Wang, H. Zhang, W. Fan, and P. Ma, "Adaptive control method for chaotic power systems based on finite-time stability theory and passivity-based control approach," Chaos, Solitons and Fractals, vol. 112, pp. 159-167, 2018.

[4] H. Liu, Y. Pan, S. Li, and Y. Chen, "Adaptive fuzzy backstepping control of fractional-order nonlinear systems," IEEE Transactions on Systems, Man, and Cybernetics: Systems, vol. 47, no. 8, pp. 2209-2217, 2017.

[5] J. Lei and L. Wang, "Backstepping synchronous control of chaotic system with reduced number of active inputs," Optik, vol. 127, no. 23, pp. 11364-11373, 2016.

[6] S. Ha, H. Liu, S. Li, and A. Liu, "Backstepping-based adaptive fuzzy synchronization control for a class of fractional-order chaotic systems with input saturation," International Journal of Fuzzy Systems, vol. 21, no. 5, pp. 1571-1584, 2019.

[7] X.-F. Li, Y.-D. Chu, A. Y. T. Leung, and H. Zhang, "Synchronization of uncertain chaotic systems via completeadaptive-impulsive controls," Chaos, Solitons and Fractals, vol. 100, pp. 24-30, 2017. 
[8] M. Zheng, L. Li, H. Peng et al., "Finite-time synchronization of complex dynamical networks with multi-links via intermittent controls," The European Physical Journal B, vol. 89, no. 2, p. 43, 2016.

[9] H. Liu, Y. Pan, S. Li, and Y. Chen, "Synchronization for fractional-order neural networks with full/under-actuation using fractional-order sliding mode control," International Journal of Machine Learning and Cybernetics, vol. 9, no. 7, pp. 1219-1232, 2018.

[10] S. Vaidyanathan and A. T. Azar, "Anti-synchronization of identical chaotic systems using sliding mode control and an application to vaidyanathan-madhavan chaotic systems," in Advances and Applications in Sliding Mode Control Systems, pp. 527-547, Springer, 2015.

[11] H. Wang, Z.-Z. Han, Q.-Y. Xie, and W. Zhang, "Finite-time chaos control via nonsingular terminal sliding mode control," Communications in Nonlinear Science and Numerical Simulation, vol. 14, no. 6, pp. 2728-2733, 2009.

[12] C.-C. Yang and C.-J. Ou, "Adaptive terminal sliding mode control subject to input nonlinearity for synchronization of chaotic gyros," Communications in Nonlinear Science and Numerical Simulation, vol. 18, no. 3, pp. 682-691, 2013.

[13] X. Chen, J. H. Park, J. Cao, and J. Qiu, “Adaptive synchronization of multiple uncertain coupled chaotic systems via sliding mode control," Neurocomputing, vol. 273, pp. 9-21, 2018.

[14] J. Wang and C. Yang, "Chaos synchronization of a finance chaotic system with an integral sliding mode controller," Journal of Mathematics, vol. 2021, Article ID 6611031, 9 pages, 2021.

[15] D. A. Haghighi and S. Mobayen, "Design of an adaptive supertwisting decoupled terminal sliding mode control scheme for a class of fourth-order systems," ISA Transactions, vol. 75, pp. 216-225, 2018.

[16] Y. Zhou, H. Liu, J. Cao, and S. Li, "Composite learning fuzzy synchronization for incommensurate fractional-order chaotic systems with time-varying delays," International Journal of Adaptive Control and Signal Processing, vol. 33, no. 12, pp. 1739-1758, 2019.

[17] A. Vahidi-Moghaddam, A. Rajaei, and M. Ayati, "Disturbance-observer-based fuzzy terminal sliding mode control for mimo uncertain nonlinear systems," Applied Mathematical Modelling, vol. 70, pp. 109-127, 2019.

[18] B. $\mathrm{Xu}$, "Composite learning finite-time control with application to quadrotors," IEEE Transactions on Systems, Man, and Cybernetics: Systems, vol. 48, no. 10, pp. 1806-1815, 2017.

[19] M. Chen and S. S. Ge, "Direct adaptive neural control for a class of uncertain nonaffine nonlinear systems based on disturbance observer," IEEE Transactions on Cybernetics, vol. 43, no. 4, pp. 1213-1225, 2012.

[20] B. Xu, Z. Shi, and C. Yang, "Composite fuzzy control of a class of uncertain nonlinear systems with disturbance observer," Nonlinear Dynamics, vol. 80, no. 1-2, pp. 341-351, 2015.

[21] M. Chen, Q. Wu, and C. Jiang, "Disturbance-observer-based robust synchronization control of uncertain chaotic systems," Nonlinear Dynamics, vol. 70, no. 4, pp. 2421-2432, 2012.

[22] B. Xu, F. Sun, Y. Pan, and B. Chen, "Disturbance observer based composite learning fuzzy control of nonlinear systems with unknown dead zone," IEEE Transactions on Systems, Man, and Cybernetics: Systems, vol. 47, no. 8, pp. 1854-1862, 2016.

[23] L. Yang and J. Yang, "Nonsingular fast terminal sliding-mode control for nonlinear dynamical systems," International
Journal of Robust and Nonlinear Control, vol. 21, no. 16, pp. 1865-1879, 2011.

[24] J. Keighobadi, M. Hosseini-Pishrobat, and J. Faraji, "Adaptive neural dynamic surface control of mechanical systems using integral terminal sliding mode," Neurocomputing, vol. 379, pp. 141-151, 2020.

[25] D. Deepika, S. Kaur, and S. Narayan, "Integral terminal sliding mode control unified with ude for output constrained tracking of mismatched uncertain non-linear systems," ISA Transactions, vol. 101, pp. 1-9, 2020.

[26] J. Jian, X. Deng, and J. Wang, "Globally exponentially attractive set and synchronization of a class of chaotic finance system," in Proceedings of the International Symposium on Neural Networks, pp. 253-261, Springer, Wuhan, China, May 2009. 